Article

\title{
The Listeria monocytogenes Key Virulence Determinants $h l y$ and $p r f A$ are involved in Biofilm Formation and Aggregation but not Colonization of Fresh Produce
}

\author{
Robert Price, Victor Jayeola, Jeffrey Niedermeyer, Cameron Parsons * ${ }^{(\mathbb{D})}$ and Sophia Kathariou \\ Department of Food, Bioprocessing and Nutrition Sciences, North Carolina State University, Raleigh, NC 27604, \\ USA; reprice2@ncsu.edu (R.P.); vjayeol@ncsu.edu (V.J.); janieder@ncsu.edu (J.N.); skathar@ncsu.edu (S.K.) \\ * Correspondence: ctparson@ncsu.edu
}

Received: 21 December 2017; Accepted: 27 January 2018; Published: 1 February 2018

\begin{abstract}
Listeria monocytogenes has been extensively studied as a model facultative intracellular pathogen. While the roles of major virulence factors in host-pathogen interactions have been extensively characterized, recent work suggests that some of these factors can also contribute to environmental proliferation of this pathogen. In this study, we characterized two non-hemolytic transposon mutants of strain 2011L-2858 (serotype 1/2b), implicated in the 2011 listeriosis outbreak via whole cantaloupe, for their capacity to form biofilms on polystyrene, aggregate, and colonize cantaloupe rind. One mutant harbored a single mariner-based transposon insertion in $h l y$, encoding the hemolysin Listeriolysin $\mathrm{O}$, while the other harbored a single insertion in $\operatorname{prf} A$, encoding PrfA, a master regulator for $h l y$ and numerous other virulence genes. Biofilm formation was significantly reduced in the prfA mutant, and to a lesser extent, in the hly mutant. Inactivation of either hly or prfA significantly reduced L. monocytogenes aggregation. However, both mutants adhered similarly to the wildtype parental strain on cantaloupe rind at either 25 or $37^{\circ} \mathrm{C}$. Furthermore, growth and competitive fitness of the mutants on cantaloupe rind was not significantly impacted at either temperature. The findings suggest that, in spite of their involvement in biofilm formation and aggregation, these key virulence determinants may not be required for the ability of L. monocytogenes to colonize fresh produce.
\end{abstract}

Keywords: Listeria monocytogenes; biofilm; aggregation; produce

\section{Introduction}

Listeria monocytogenes is a facultative intracellular pathogen responsible for human foodborne disease (listeriosis) associated with severe symptoms (e.g., septicemia, meningitis, stillbirths) and high case fatality rate [1,2]. Strains implicated in human listeriosis are primarily of three serotypes, $1 / 2 \mathrm{a}$, $1 / 2 b$ and $4 b[3,4]$. Even though foods such as soft cheeses, deli meats and processed seafood have been implicated in numerous outbreaks, in recent years produce has been increasingly recognized as a vehicle for human listeriosis [5,6]. In 2011, a large, multistate listeriosis outbreak in the United States was attributed to whole cantaloupe contaminated with strains of serotype $1 / 2 \mathrm{a}$ and $1 / 2 \mathrm{~b}$. The outbreak resulted in 147 reported cases of listeriosis and multiple deaths. This was the first listeriosis outbreak involving whole cantaloupe and the largest reported to date [7].

The L. monocytogenes hemolysin Listeriolysin O (LLO), encoded by hly, and PrfA, a master transcriptional activator for $h l y$ and numerous other virulence genes, are key virulence determinants of L. monocytogenes, extensively characterized for their roles in host-pathogen interactions [8-11]. However, PrfA has also been found to be required for biofilm formation by the serotype $1 / 2$ a 
strains 10403S and EGD [12,13], and mutants with deregulated, constitutive $\operatorname{prfA}$ expression ("PrfA*") exhibited decreased competitive fitness upon exposure to several environmental stresses [14]. In addition, ActA, a virulence determinant under PrfA control and key to intracellular motility and cell-to-cell spread of L. monocytogenes, was shown to be required for L. monocytogenes EGD cell aggregation and biofilm formation [13]. Even though underlying mechanisms remain to be fully elucidated, such findings suggest that these virulence determinants also contribute to environmental adaptation of L. monocytogenes outside of the animal host. However, these studies were largely performed with reference strains such as $10403 \mathrm{~S}$ and EGD which belong to clonal groups that seem to be under-represented in human disease [15] and may not fully reflect adaptive attributes of strains frequently implicated in listeriosis outbreaks, considering the increasingly important role of fresh produce in listeriosis outbreaks [5,6], similar studies with strains from produce-related outbreaks have been noticeably lacking. Potential links between prfA or key virulence determinants under PrfA control and the capacity of L. monocytogenes to colonize fresh produce also remain to be identified and investigated. The objective of the current study was to characterize the impact of prfA and hly on the ability of L. monocytogenes strain 2011L-2858, a serotype $1 / 2 \mathrm{~b}$ strain associated with a major listeriosis outbreak involving contaminated whole cantaloupe in 2011, to form biofilms, aggregate, and colonize the surface of fresh cantaloupe.

\section{Results and Discussion}

The serotype $1 / 2 \mathrm{~b}$ strain $2011 \mathrm{~L}-2858$, hereafter referred to as " 2858 ", belongs to clonal complex 5, and was implicated in the 2011 multistate outbreak of listeriosis [5,16]. Isolates of the same serotype and genotype contributed to $27 \%$ of the cases and $30 \%$ of the fatalities in this outbreak and were also recovered from whole fruit and the packing environment [5,7]. Screening of approx. 1900 transposon mutants of this strain on blood agar plates revealed three that were non-hemolytic. Among them, mutants B2G6 (insertion at nt 225 of $h l y$ ) and J2E3 (insertion at nt 65 of $p r f A$ ) were chosen for further work. Southern blots confirmed that each mutant harbored a single insertion of the transposon (data not shown). The mutants were indistinguishable from the parental strain and each other in motility (data not shown), colony or cell morphology, and growth rate in BHI at 28 or $37^{\circ} \mathrm{C}$ (Supplemental Figures S1-S3).

\section{1. hly and prfA are Required for Biofilm Formation of L. monocytogenes}

In spite of increasing interest in the interface between environmental biology and pathogenesis of L. monocytogenes $[9,10,17-19]$ the potential roles of key virulence genes of L. monocytogenes in adaptations other than those directly associated with pathogenesis remain poorly understood. Previous studies with L. monocytogenes reference strains $10403 S$ and EGD (both of serotype 1/2a) revealed a significant requirement of prfA for biofilm formation in polyvinyl 96-well plates (Lemon, Freitag and Kolter 2010; Travier et al., 2013). However, hly mutants of strain $10403 \mathrm{~S}$ did not exhibit defects in biofilm formation [12], while hly inactivation of strain EGD resulted in moderate biofilm reductions [13]. In the case of strain 2858, both the $\operatorname{prf} A$ and hly deficient mutants exhibited significant $(p<0.05)$ reduction in biofilm (Figure 1). Inactivation of $\operatorname{prf} A$ was accompanied by a pronounced reduction in biofilm while the hly mutant exhibited moderate reduction, similarly to what was observed with strain EGD [13]. Such data suggest that, besides $h l y$, additional determinants under PrfA transcriptional control are implicated in biofilm formation. 


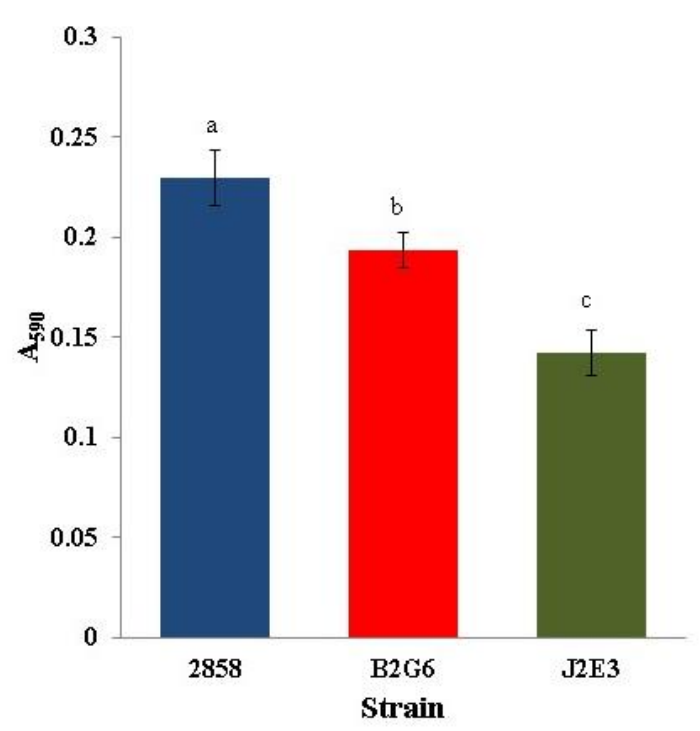

Figure 1. Impact of $h l y$ and prfA on biofilm formation. Biofilms were established at $37^{\circ} \mathrm{C}$ in wells of 96-well polystyrene plates over $48 \mathrm{~h}$ (8 wells/strain) and measured following staining with crystal violet as described in Materials and Methods. Strains from this study are shown on the $\mathrm{x}$ axis, wild type 2011L-2858 (2858), hly inactivated mutant B2G6, and prfA inactivated mutant J2E3. The y axis indicates absorbance of ethanol used to destain crystal violet stained wells at $590 \mathrm{~nm}$, thus serving as an indication of biofilm formation. Different letters indicate statistically significant differences $(p<0.05)$. Data are from three independent trials.

The master virulence regulator PrfA controls expression of several virulence genes, including hly and $a c t A$, with the latter having been shown to contribute significantly to L. monocytogenes aggregation and biofilm formation [13]. Such involvement of ActA on biofilm formation, together with the moderate impacts of hly inactivation observed here and by others [13], may contribute to the much more pronounced biofilm reductions exhibited by the prfA mutant J2E3 than by the hly mutant B2G6 (Figure 1).

\subsection{Inactivation of Either prfA or hly Results in Decreased Aggregation}

Aggregation in L. monocytogenes in vitro has been shown to require intact prfA; in a murine model it is associated with aggregation and persistence in the intestinal lumen and impacts the levels and length of L. monocytogenes shedding in feces [13]. Aggregation assays in our study showed that prfA inactivation significantly decreased cell aggregation in vitro at $37^{\circ} \mathrm{C}$ (Figures 2 and 3), in agreement with previous reports with strain EGD [13]. The differences in $\mathrm{OD}_{600}$ (Figure 2), which suggested differences in aggregative ability between wild type 2858 and both mutant strains were corroborated by phase contrast microscopy (Figure 3) which also revealed more wild type formed aggregates than either of the mutant strains. In strain EGD, the PrfA-controlled determinant ActA was shown to mediate aggregation via direct ActA-ActA interactions in the C-terminal region of the protein which is not responsible for actin polymerization and intracellular motility during infection [13]. Similar ActA-based interactions likely contribute to the role of $\operatorname{prf} A$ in aggregation of strain 2858, though analysis of $a c t A$ mutants will be required for confirmation. 


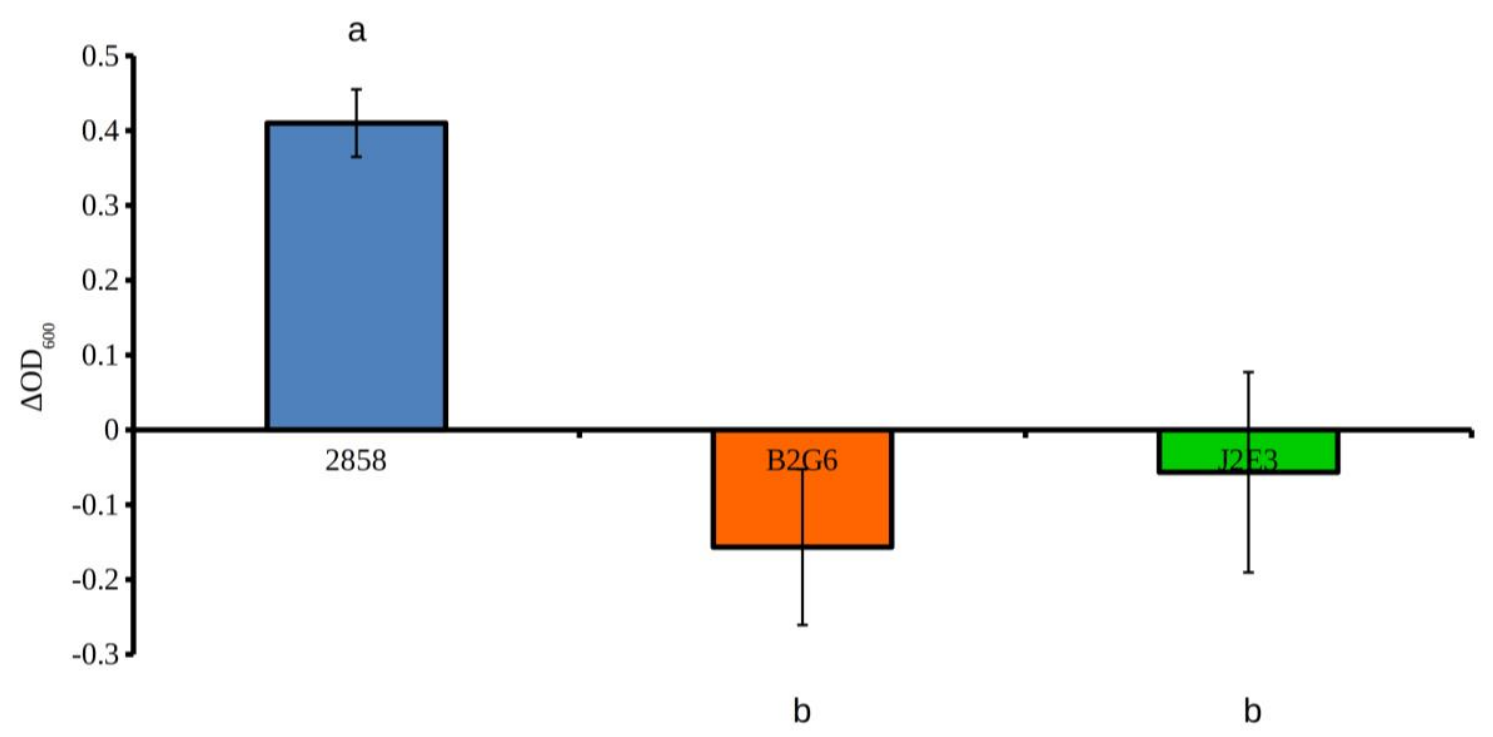

Figure 2. Impact of inactivation of either hly or $\operatorname{prf} A$ on cell aggregation. Aggregation assays of 2858 (wild type parental strain), hly mutant B2G6 and prfA mutant J2E3 were performed in BHI as described in Materials and Methods. Different letters indicate statistically significant differences $(p<0.05)$.

A.

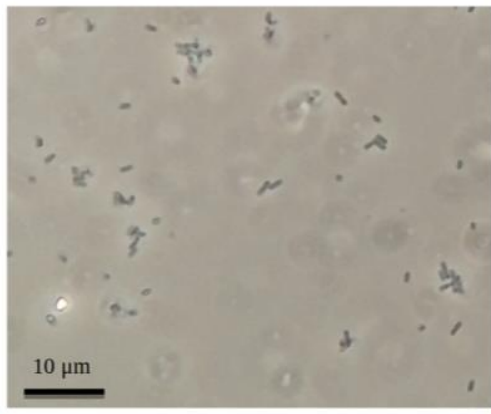

B.



C.

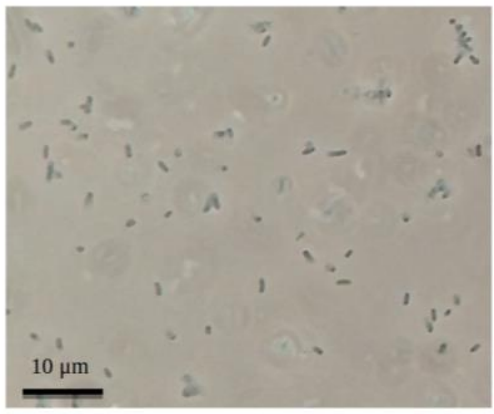

Figure 3. L. monocytogenes aggregation. Aggregation assays were performed in $\mathrm{BHI}$ at $37^{\circ} \mathrm{C}$, and bacteria from approx. $1 \mathrm{~cm}$ below the surface were visualized with phase contrast microscopy as described in Materials and Methods. (A) Parental strain 2858; (B) hly mutant B2G6; (C) prfA mutant J2E3.

Surprisingly, inactivation of hly also had a pronounced impact on aggregation in strain 2858 (Figures 2 and 3). This contrasted with strain EGD, where hly had only marginal involvement in aggregation [13]. The more noticeable involvement of $h l y$ in aggregation of strain 2858 in the current study may reflect strain-specific differences which remain to be characterized. In addition to having different serotypes, strains EGD and 2858 belong to different lineages (lineage II and I, respectively), and are thus likely to differ in multiple attributes. Molecular mechanisms mediating the role of LLO in aggregation in strain 2858 remain to be identified. It also remains to be determined whether $h l y$ has generally a more pronounced impact on aggregation in serotype $1 / 2 b$ than serotype $1 / 2 a$, or in lineage I vs. lineage II. Lastly, the aggregation impact of the prfA mutation on other determinants besides hly that are under PrfA control remains to be determined in this strain. 
2.3. Insertional Inactivation of hly or prfA does not Impact Growth and Adherence of L. monocytogenes on Cantaloupe Rind at 25 or $37^{\circ} \mathrm{C}$

The apparent requirement of intact prfA and $h l y$ for biofilm formation suggested the possibility that colonization of produce might also be impaired in the mutants. Since expression of hly and prfA is under temperature control and is optimal at $37^{\circ} \mathrm{C}[10,20]$, we investigated produce colonization both at $25^{\circ} \mathrm{C}$, a temperature relevant for produce contamination, and at $37^{\circ} \mathrm{C}$. The parental strain and the two mutants showed similar adherence on cantaloupe rind at both 25 and $37^{\circ} \mathrm{C}$. For all three strains, adherent cells represented $35 \pm 10 \%$ of the population inoculated on the rind. Furthermore, no significant differences in growth on the cantaloupe rind were noted between the parental strain 2858 and either B2G6 or J2E3 at $25^{\circ} \mathrm{C}$ (monitored up to $72 \mathrm{~h}$ ) (Figure 4A). Growth was also not impaired at $37^{\circ} \mathrm{C}$ (Figure $4 \mathrm{~B}$ ), a temperature at which, as indicated above, $\operatorname{prf} A$ and $h l y$ would be expected to be optimally expressed $[10,20]$.
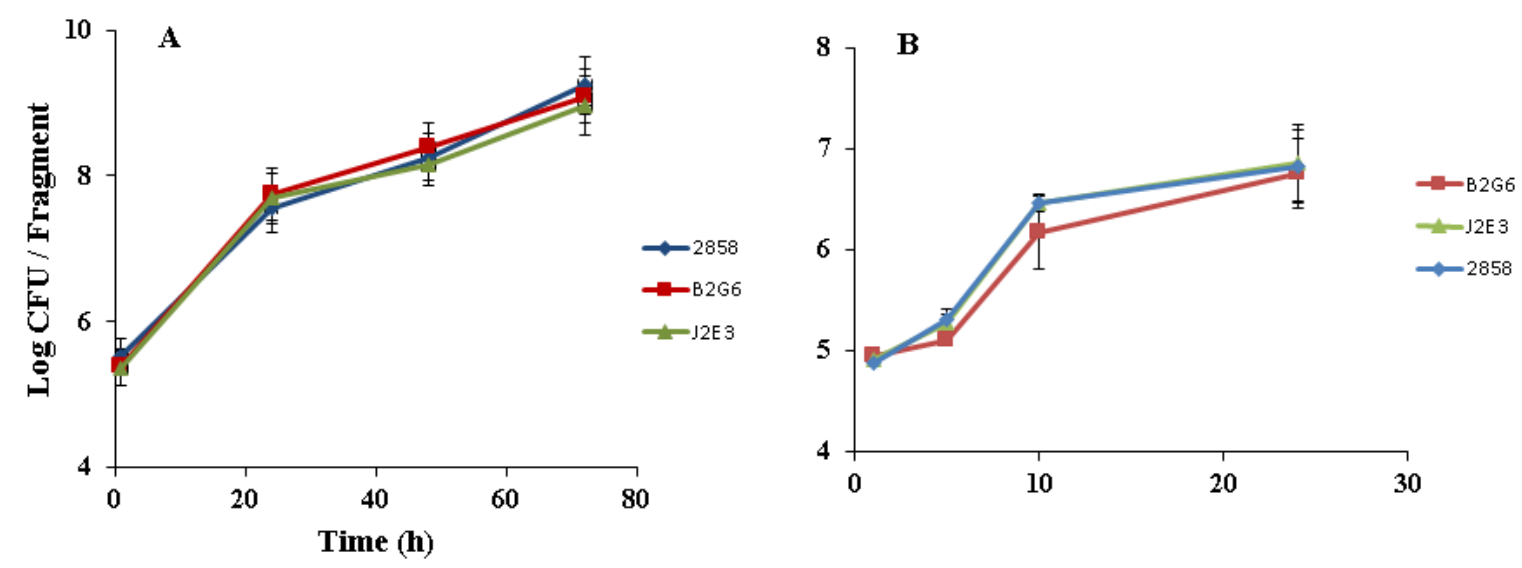

Figure 4. Growth of hly and $\operatorname{prf} A$ mutants on cantaloupe rind. Rind fragments were inoculated with approx. $10^{5} \mathrm{CFU}$ of each strain and growth was determined at (A) $25^{\circ} \mathrm{C}$ and (B) $37^{\circ} \mathrm{C}$ as described in Materials and Methods. Data are from three independent trials, each done in duplicate.

To determine potential impacts of the mutations on the relative fitness of the bacteria on cantaloupe, the rind was inoculated with 1:1 mixtures of the parental strain 2858 and each of the mutants, and incubated at $25^{\circ} \mathrm{C}$ (up to $72 \mathrm{~h}$ ) and $37^{\circ} \mathrm{C}(24 \mathrm{~h}$ ). Screening of colonies from the MOX plates on media with and without erythromycin revealed that for both mutants the ratio of parental strain to mutant remained around $50 \%$ at all tested time points (Figure 5). Similar data were obtained when randomly-selected colonies from the MOX plates were patched onto blood agar and incubated at $37^{\circ} \mathrm{C}$ (data not shown). Such data suggest that relative fitness of the hly or the prfA mutant on the cantaloupe rind at either 25 or $37^{\circ} \mathrm{C}$ did not differ significantly from that of the parental strain.

Surface-specific differences may underlie the observed lack of correlation between biofilm formation and colonization of living plant tissue. Cellulose on the latter, for instance, may facilitate binding of L. monocytogenes, e.g., through the Listeria cellulose binding protein, Lcp [21]. A similar lack of correlation was found with the L. monocytogenes cadmium resistance determinant cadA4, which was implicated in biofilm formation but not in colonization of produce [19], and the L. monocytogenes penicillin-binding protein $p b p 4$ [22]. In addition to the composition of the surface, the cantaloupes were all bought at retail and retained their natural microbiota. Interactions with the background microbiota or their metabolites may also have impacted the ability of L. monocytogenes to form biofilms. 
A

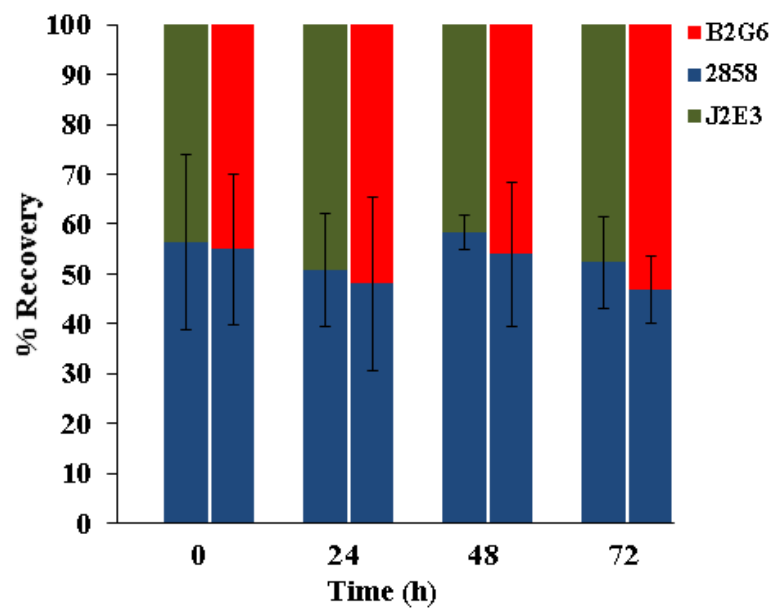

$\mathbf{B}$

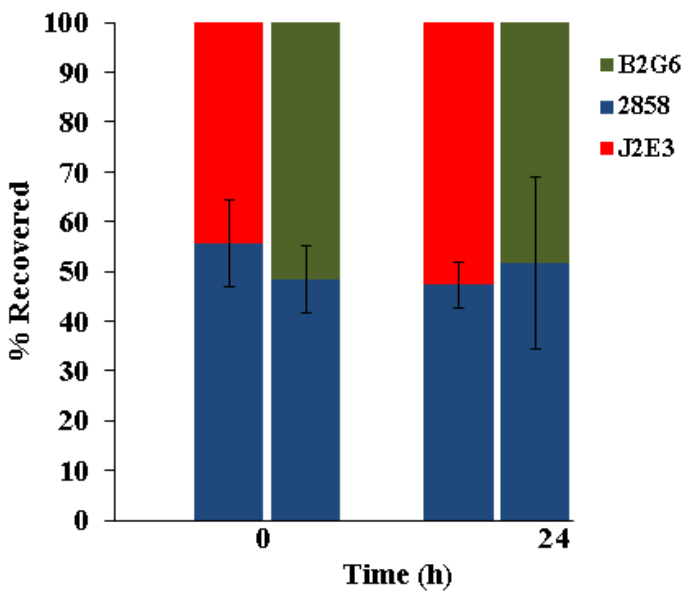

Figure 5. Competitive fitness of hly and prfA mutants on cantaloupe rind. Cantaloupe fragments were inoculated with 1:1 mixtures of the wildtype parental strain 2858 and each mutant, and inoculated fragments were incubated at (A) $25^{\circ} \mathrm{C}$ and (B) $37^{\circ} \mathrm{C}$. Ratios were determined by sub-culturing colonies from MOX plates in individual wells of 96 -well plates and subsequent differentiation on media with and without erythromycin, as described in Materials and Methods. Time 0 corresponds to 30 min after inoculation. Data are from three independent trials, each done in duplicate.

In conclusion, our analysis of non-hemolytic mutants of strain 2858 , a serotype $1 / 2 b$ strain that contributed to the 2011 listeriosis outbreak via whole cantaloupe, revealed that prfA and, to a lesser extent, $h l y$, both contribute to the ability of L. monocytogenes to form biofilms, and both determinants are involved in aggregation of L. monocytogenes. These virulence genes may therefore play important roles in environmental contamination of the produce-packing facilities and food production equipment, thus contributing to food safety risks. However, under the employed conditions neither $\operatorname{prf} A$ nor $h l y$ were found to be required for adherence, growth or competitive fitness of L. monocytogenes on the cantaloupe rind. Further studies of this and other strains are needed to more fully characterize the involvement of these and other virulence factors in environmental survival and persistence of L. monocytogenes, and in contamination of food by this troublesome pathogen.

\section{Materials and Methods}

\subsection{Bacterial Strains and Growth Conditions}

The serotype $1 / 2$ b strain $2011 \mathrm{~L}-2858$, referred to here as " 2858 ", was kindly obtained from Cheryl Tarr and was previously investigated for its potential to adhere and grow on cantaloupe [23], as well as the role of a penicillin-binding protein encoded by $p b p 4$ on copper tolerance and virulence [22]. Strains B2G6 and J2E3 are non-hemolytic mutants of strain 2858 obtained as described below from screening a mariner-based mutant library on blood agar (Remel Inc., Lenexa, KS, USA) plates. Unless otherwise indicated, L. monocytogenes was grown at $37^{\circ} \mathrm{C}$ in brain heart infusion (BHI) broth (Becton, Dickinson \& Co., Sparks, MD, USA) or on BHI agar (BHIA, BHI with 1.2\% Bacto-agar, Becton, Dickinson \& Co.). When needed, erythromycin (MP Biomedicals, Solon, OH, USA) was added at $5 \mu \mathrm{g} / \mathrm{mL}$ in BHI (BHI-Em5) or in BHIA (BHIA-Em5) and kanamycin (Fisher Scientific, Fair Lawn, NJ, USA) was added at $10 \mu \mathrm{g} / \mathrm{mL}$ to BHI (BHI-Km10) and BHIA (BHIA-Km10).

\subsection{Mutant Library Construction, Screening for Non-Hemolytic Mutants, Determination of Transposon Copy Number and Localization}

A mariner-based transposon mutant library (approx. 1900 mutants) of strain 2858 was constructed as described using pMC38 $[19,24]$. Mutants that were confirmed to be erythromycin-resistant 
(as expected by presence of the transposon) and kanamycin-sensitive (as expected upon loss of the plasmid vector for the transposon) were individually screened on blood agar (Remel Inc., Lenexa, $\mathrm{KS}, \mathrm{USA}$ ) using a sterile 48 -pin stainless steel replicator. The plates were incubated at $37^{\circ} \mathrm{C}$ for $36 \mathrm{~h}$ and examined for hemolysis zones typical of L. monocytogenes. The number of transposon insertions in each mutant was determined by Southern blot as described $[19,24]$. To identify the sites of transposon insertion, arbitrary PCR was performed as described [19,24]. PCR products were sequenced (Genewiz Inc., South Plainfield, NJ, USA), and sequences were analyzed by nucleotide BLAST.

\subsection{Biofilm Formation and Aggregation Assessments}

Procedures previously described [19] were employed to assess biofilm formation in 96-well polystyrene plates (Greiner Bio-One, VWR, Suwanee, GA, USA). Briefly, L. monocytogenes strains were grown in wells of 96 -well plates at $37^{\circ} \mathrm{C}$ for $48 \mathrm{~h}$ without agitation. Upon removal of the supernatant, each well was washed three times with deionized water and allowed to air-dry for approx. $45 \mathrm{~min}$. Crystal violet staining, solubilization of the dye in $95 \%$ ethanol and biofilm measurements via $A_{590}$ determinations were as described [19]. L. monocytogenes was grown in eight wells/strain for each trial, and analyses were done in at least three independent trials. Aggregation assessments were carried out at $37^{\circ} \mathrm{C}$ as described previously [13]. Aggregation was calculated by subtracting $\mathrm{OD}_{600}$ at $24 \mathrm{~h}$ from the $\mathrm{OD}_{600}$ at the start of the assay. For microscopy, material from approx. $1 \mathrm{~cm}$ below the surface was carefully removed and cells visualized by phase contrast microscopy on a Leica microscope (model LMB 2).

\subsection{Cantaloupe Rind Adherence and Growth Assessments}

Cantaloupes were purchased from local grocery stores in Raleigh, NC and $2 \times 2 \times 0.5 \mathrm{~cm}$ rind fragments were obtained as described [23]. Preparation of inoculum and spot-inoculation of the fragments with 10 evenly separated droplets of $10 \mu \mathrm{L}$ each (total inoculum, $100 \mu \mathrm{L}$ ) were as described [23]. Adherence and growth assays were also as described [23]. Briefly, inoculated fragments were incubated at the indicated temperature for $1 \mathrm{~h}$, loosely adherent cells were removed by gentle washes, and tightly adhered cells were then removed by vortexing and enumerated by plating dilutions of the rinsate on modified Oxford Listeria selective agar (MOX; Oxoid, Hampshire, UK) and incubating at $37^{\circ} \mathrm{C}$ for $48 \mathrm{~h}$. To assess growth, petri dishes with inoculated rind fragments were sealed with parafilm and stored for up to $72 \mathrm{~h}$ at room temperature $\left(25^{\circ} \mathrm{C}\right)$ or up to $24 \mathrm{~h}$ at $37^{\circ} \mathrm{C}$. At specific time points, rinsates of two inoculated cantaloupe fragments per strain were diluted and plated on MOX, followed by incubation for $48 \mathrm{~h}$ at $37^{\circ} \mathrm{C}$. Each assessment was done in duplicate, and in at least three independent trials.

\subsection{Competitive Fitness Assessments}

Inoculum for mixed-strain inoculations (1:1 mixture of the wild type parental strain 2858 and each mutant) was prepared by combining cell suspensions corresponding to equal CFUs of each strain. Randomly-chosen colonies from the MOX plates were inoculated individually into 96-well plates containing $200 \mu \mathrm{L} \mathrm{BHI}$ and incubated for approx. $16 \mathrm{~h}$ at $37^{\circ} \mathrm{C}$. The 96 -well cultures were then stamped using a sterile 48-pin replicator onto BHIA and BHIA-Em5. The ratio of parental strain 2858 (erythromycin-susceptible) and mutant (erythromycin-resistant) was determined following $36 \mathrm{~h}$ of incubation at $37^{\circ} \mathrm{C}$. To confirm these findings, randomly-selected colonies from the MOX plates were also streaked on blood agar (Remel, Inc.). The plates were incubated at $37^{\circ} \mathrm{C}$ for $36 \mathrm{~h}$ and the ratio of hemolytic and non-hemolytic cultures was determined. Each competitive fitness assay was done in at least three independent trials.

\subsection{Statistical Analysis}

For statistical analysis of growth and adherence on produce, including growth in competitive fitness experiments, one-way analysis of variance (ANOVA) with a Tukey's test was used at $p<0.05$, 
with SPSS version 22 (IBM Corporation Software Group, Somers, NY, USA). Biofilm and aggregation data were analyzed using a paired Student's t-test $(p<0.05)$.

Supplementary Materials: The following are available online at www.mdpi.com/2076-0817/7/1/18/s1, Figure S1: Colony morphology of (A) wild type 2858, (B) hly inactivated mutant B2G6, and (C) prfA inactivated mutant J2E3; Figure S2: Cell morphology of (A) wild type 2858, (B) hly inactivated mutant B2G6, and (C) prfA inactivated mutant J2E3; Figure S3 Growth curves of wild type 2858, hly inactivated mutant B2G6, and prfA inactivated mutant J2E3 at (A) 28 and (B) $37^{\circ} \mathrm{C}$.

Acknowledgments: This study was partially funded by USDA grant 2011-67017-30218. We thank Fred Breidt for feedback and access to equipment utilized in the study. We appreciate the contributions of Mira Rakic-Martinez, Daniela Lopez and Ben Costollo. We thank all other members of our laboratory for their support.

Author Contributions: RP and VJ implemented adherence, growth and competitive fitness assays, and contributed to the preparation of the manuscript. JN obtained biofilm and aggregation data and contributed to manuscript figures and editing. CP constructed mutant library, isolated and characterized the mutants, and contributed to manuscript preparation. SK oversaw project design and contributed to manuscript preparation.

Conflicts of Interest: The authors declare no conflict of interest.

\section{References}

1. Painter, J.; Slutsker, L. Listeriosis in humans. In Listeria, Listeriosis and Food Safety; CRC Press: Boca Raton, FL, USA, 2007; Volume 30, pp. 85-109, ISBN 978-0-8247-5750-2.

2. Scallan, E.; Hoekstra, R.M.; Angulo, F.J.; Tauxe, R.V.; Widdowson, M.A.; Roy, S.L.; Jones, J.L.; Griffin, P.M. Foodborne illness acquired in the United States-major pathogens. Emerg. Infect. Dis. 2011, 17, 7-15. [CrossRef] [PubMed]

3. Kathariou, S. Listeria monocytogenes virulence and pathogenicity, a food safety perspective. J. Food Prot. 2002, 65, 1811-1829. [CrossRef] [PubMed]

4. Swaminathan, B.; Gerner-Smidt, P. The epidemiology of human listeriosis. Microbes Infect. 2007, 9, $1236-1243$. [CrossRef] [PubMed]

5. Garner, D.; Kathariou, S. Fresh produce-associated listeriosis outbreaks, sources of concern, teachable moments, and insights. J. Food Prot. 2016, 79, 337-344. [CrossRef] [PubMed]

6. Cartwright, E.J.; Jackson, K.A.; Johnson, S.D.; Graves, L.M.; Silk, B.J.; Mahon, B.E. Listeriosis outbreaks and associated food vehicles, United States, 1998-2008. Emerg. Infect. Dis. 2013, 19, 1-9. [CrossRef] [PubMed]

7. McCollum, J.T.; Cronquist, A.B.; Silk, B.J.; Jackson, K.A.; O'Connor, K.A.; Cosgrove, S.; Gossack, J.P.; Parachini, S.S.; Jain, N.S.; Ettestad, P.; et al. Multistate outbreak of listeriosis associated with cantaloupe. N. Engl. J. Med. 2013, 369, 944-953. [CrossRef] [PubMed]

8. Gray, M.J.; Freitag, N.E.; Boor, K.J. How the bacterial pathogen Listeria monocytogenes mediates the switch from environmental Dr. Jekyll to pathogenic Mr. Hyde. Infect. Immun. 2006, 74, 2506-2512. [CrossRef] [PubMed]

9. Freitag, N.E.; Port, G.C.; Miner, M.D. Listeria monocytogenes-From saprophyte to intracellular pathogen. Nat. Rev. Microbiol. 2009, 7, 623-628. [CrossRef] [PubMed]

10. De las Heras, A.; Cain, R.J.; Bielecka, M.K.; Vazquez-Boland, J.A. Regulation of Listeria virulence: PrfA master and commander. Curr. Opin. Microbiol. 2011, 14, 118-127. [CrossRef] [PubMed]

11. Hamon, M.A.; Ribet, D.; Stavru, F.; Cossart, P. Listeriolysin O: The Swiss army knife of Listeria. Trends Microbiol. 2012, 20, 360-368. [CrossRef] [PubMed]

12. Lemon, K.P.; Freitag, N.E.; Kolter, R. The virulence regulator PrfA promotes biofilm formation by Listeria monocytogenes. J. Bacteriol. 2010, 192, 3969-3976. [CrossRef] [PubMed]

13. Travier, L.; Guadagnini, S.; Gouin, E.; Dufour, A.; Chenal-Francisque, V.; Cossart, P.; Olivo-Marin, J.C.; Ghigo, J.M.; Disson, O.; Lecuit, M. ActA promotes Listeria monocytogenes aggregation, intestinal colonization and carriage. PLoS Pathog. 2013, 9. [CrossRef] [PubMed]

14. Xayarath, B.; Freitag, N.E. Optimizing the balance between host and environmental survival skills: Lessons learned from Listeria monocytogenes. Future Microbiol. 2012, 7, 839-852. [CrossRef] [PubMed]

15. Maury, M.M.; Tsai, Y.-H.; Charlier, C.; Touchon, M.; Chenal-Francisque, V.; Leclercq, A.; Criscuolo, A.; Gaultier, C.; Roussel, S.; Brisabois, A.; et al. Uncovering Listeria monocytogenes hypervirulence by harnessing its biodiversity. Nat. Genet. 2016. [CrossRef] [PubMed] 
16. Cantinelli, T.; Chenal-Francisque, V.; Diancourt, L.; Frezal, L.; Leclercq, A.; Wirth, T.; Lecuit, M.; Brisse, S. “Epidemic clones" of Listeria monocytogenes are widespread and ancient clonal groups. J. Clin. Microbiol. 2013, 51, 3770-3779. [CrossRef] [PubMed]

17. Pombinho, R.; Camejo, A.; Vieira, A.; Reis, O.; Carvalho, F.; Almeida, M.T.; Pinheiro, J.C.; Sousa, S.; Cabanes, D. Listeria monocytogenes CadC regulates cadmium efflux and fine-tunes lipoprotein localization to escape the host immune response and promote infection. J. Infect. Dis. 2017, 215, 1468-1479. [CrossRef] [PubMed]

18. Lebreton, A.; Stavru, F.; Brisse, S.; Cossart, P. 1926-2016: 90 Years of listeriology. Microbes Infect. 2016, 18, 711-723. [CrossRef] [PubMed]

19. Parsons, C.; Lee, S.; Jayeola, V.; Kathariou, S. Novel cadmium resistance determinant in Listeria monocytogenes. Appl. Environ. Microbiol. 2017, 83, e02580-16. [CrossRef] [PubMed]

20. Johansson, J.; Mandin, P.; Renzoni, A.; Chiaruttini, C.; Springer, M.; Cossart, P. An RNA thermosensor controls expression of virulence genes in Listeria monocytogenes. Cell 2002, 110, 551-561. [CrossRef]

21. Bae, D.; Seo, K.S.; Zhang, T.; Wang, C. Characterization of a potential Listeria monocytogenes virulence factor associated with attachment to fresh produce. Appl. Environ. Microbiol. 2013, 79, 6855-6861. [CrossRef] [PubMed]

22. Parsons, C.; Costolo, B.; Brown, P.; Kathariou, S. Penicillin-binding protein encoded by pbp4 is involved in mediating copper stress in Listeria monocytogenes. FEMS Microbiol. Lett. 2017, 364. [CrossRef] [PubMed]

23. Rakic-Martinez, M.; Osborne, J.; Jayeola, V.O.; Katic, V.; Kathariou, S. Capacity of Listeria monocytogenes strains from the 2011 cantaloupe outbreak to adhere, survive, and grow on cantaloupe. J. Food Prot. 2016, 79, 757-763. [CrossRef] [PubMed]

24. Cao, M.; Bitar, A.P.; Marquis, H. A mariner-based transposition system for Listeria monocytogenes. Appl. Environ. Microbiol. 2007, 73, 2758-2761. [CrossRef] [PubMed]

(C) 2018 by the authors. Licensee MDPI, Basel, Switzerland. This article is an open access article distributed under the terms and conditions of the Creative Commons Attribution (CC BY) license (http:/ / creativecommons.org/licenses/by/4.0/). 